Published in final edited form as:

Am J Perinatol. 2018 December ; 35(14): 1352-1357. doi:10.1055/s-0038-1627095.

\title{
Stage I Twin-Twin Transfusion Syndrome: Outcomes of Expectant Management and Prognostic Features
}

\author{
Erin E. Washburn, MD¹, Teresa N. Sparks, MD¹, Kristen A. Gosnell, RN, MS², Larry Rand, \\ $\mathbf{M D}^{1,2}$, Juan M. Gonzalez, MD, $\mathbf{P h D}^{1,2}$, and Vickie A. Feldstein, $\mathbf{M D}^{2,3}$ \\ ${ }^{1}$ Department of Obstetrics, Gynecology, and Reproductive Sciences, University of California San \\ Francisco, San Francisco, California \\ ${ }^{2}$ Fetal Treatment Center, University of California San Francisco, San Francisco, California \\ ${ }^{3}$ Department of Radiology and Biomedical Imaging, University of California San Francisco, San \\ Francisco, California
}

\begin{abstract}
Objective-This article describes the natural history of stage I twin-twin transfusion syndrome (TTTS) including risk of progression to higher stage TTTS and pregnancy outcomes, and to identify risk factors for progression.
\end{abstract}

Study Design—Retrospective cohort study of monochorionic diamniotic (MCDA) twin pregnancies from 2006 to 2016 with expectantly managed Quintero stage I TTTS.

\begin{abstract}
Results-A total of 30 MCDA twin pregnancies with expectantly managed stage I TTTS were identified. Of these, eight (26.7\%) progressed to higher stage TTTS. Median gestational age (GA) at diagnosis for those that progressed was $18.9 \pm 2.9$ weeks versus $21.4 \pm 3.4$ weeks in those that remained stable $(p=0.06)$. Presence of an arterioarterial (A-A) anastomosis was assessed for 20/30 patients, and eight A-A were identified. Of those, 7/8 (87.5\%) remained stable. In the complete cohort, a tiny or transiently visible bladder was noted in seven donor twins. Of these, 4/7 (57.1\%) progressed. Excluding one termination, $47 / 58$ (81.0\%) fetuses survived.
\end{abstract}

Conclusion-With expectant management of stage I TTTS, nearly three-fourths of pregnancies remain stable and most have survival of $\geq 1$ twin. A-A anastomoses were not associated with progression to higher stage TTTS, whereas earlier GA at diagnosis or a small or intermittently visible donor bladder may herald greater risk of progression.

\section{Keywords}

twin-twin transfusion syndrome; arterioarterial anastomosis; natural history; expectant management; Quintero stage I

\footnotetext{
Address for correspondence: Erin E. Washburn, MD, Department of Obstetrics, Gynecology, and Reproductive Sciences, University of California San Francisco, 550 16th Street, 7th Floor, Box 0132, San Francisco, CA 94143 (Erin.Washburn@ucsf.edu).

Note

This study was presented in poster format at the 37th Annual Pregnancy Meeting of the Society for Maternal Fetal Medicine in Las Vegas, NV on January 23-27, 2017.

Conflict of Interest

None.
} 
Twin-twin transfusion syndrome (TTTS) affects 8 to $10 \%$ of all monochorionic pregnancies ${ }^{1,2}$ and is a major source of neonatal morbidity and mortality for monochorionic diamniotic (MCDA) twins. ${ }^{2,3}$ Untreated, severe TTTS is associated with a 70 to $100 \%$ fetal loss rate. ${ }^{3,4}$ Fetoscopic laser coagulation (FLC) of intertwin placental arteriovenous (A-A) anastomoses has been shown to improve neonatal survival and reduce rates of neurologic morbidity in higher stage TTTS $^{5-8}$ and is generally accepted as the standard of care for Quintero stage II to IV TTTS. ${ }^{9}$

The optimal management of stage I TTTS is less clear though, and treatment approaches range from expectant management to FLC. Randomized data of laser treatment for stage I TTTS is extremely limited, and no randomized data of expectant management of stage I TTTS currently exist. Non-randomized studies of the natural history of TTTS have shown that the majority of pregnancies with stage I TTTS (85\%) do not progress and have associated high neonatal survival rates. ${ }^{9-12}$ Therefore, expectant management of stage I TTTS has been considered an acceptable treatment approach. ${ }^{9,12}$

This approach was recently challenged, however, by a retrospective study from the North American Fetal Therapy Network (NAFTNET) ${ }^{13}$ that compared outcomes of expectant management, amnioreduction, and FLC for stage I TTTS. This study found significantly worse outcomes for expectant management of stage I TTTS with 34/49 (69\%) progressing in stage or suffering dual loss from spontaneous previable preterm birth. Additionally, both amnioreduction and FLC reduced the chance of dual loss as compared with expectant management, and FLC was protective against poor obstetric outcome (defined as double fetal demise or delivery < 26 weeks' gestational age [GA]). ${ }^{13}$

Given this lack of consensus regarding risk of progression and optimal management strategy for stage I TTTS, we aimed to describe the natural history of stage I TTTS including risk of progression to higher stage TTTS and pregnancy outcomes, as well as to identify risk factors for progression. Our hypotheses were that the rate of progression from stage I TTTS to higher stage TTTS would be low and obstetric outcomes of expectant management would be comparable to those following fetal intervention.

\section{Methods}

This was a retrospective cohort study of all cases of MCDA twin pregnancies referred to the University of California San Francisco (UCSF) Fetal Treatment Center (FTC) for possible TTTS from January 2006 to June 2016. Cases were identified from the FTC database. Inclusion criteria were MCDA twin pregnancies diagnosed with stage I TTTS at 16 to 28 weeks' GA, for which expectant management of stage I TTTS was pursued as is standard practice at our institution. Exclusion criteria were primary fetal intervention (amnioreduction or FLC) at the time of diagnosis of stage I TTTS, elective pregnancy termination in the absence of progression to higher stage TTTS, critically abnormal Doppler studies at diagnosis, and major fetal anomalies. Cases were also excluded if there was insufficient follow-up to determine the primary study outcome. 
The primary outcome was progression to higher stage TTTS, meaning stage II or above. Obstetric outcomes (neonatal survival and GA at delivery) and possible sonographic risk factors for progression (deepest vertical pocket [DVP] of amniotic fluid for each twin, estimated fetal weight (EFW) discordance, fetal growth restriction (FGR) of the donor twin, marginal or velamentous cord insertion of either twin, presence of visible A-A anastomosis, and markedly tiny or intermittently visible donor twin urinary bladder) were considered secondarily. For consistency and in recognition of the impact of preterm delivery on neonatal morbidity and mortality, obstetric outcomes were described in two ways as defined by Emery et $\mathrm{al}^{13}$ : number of surviving neonates to hospital discharge, and good (dual survival and delivery at $\geq 30$ weeks), mixed (single demise or delivery at 26-29.9 weeks), or poor (dual demise or delivery at $<26$ weeks).

On referral to our FTC, each pregnancy underwent ultrasound (US) evaluation including a complete anatomic survey, assessment of amniotic fluid volume by DVP, Doppler evaluation of the umbilical artery (UA) and ductus venosus (DV), and documentation of the placental cord insertion sites for both twins. TTTS was diagnosed and staged based on the Quintero staging system, ${ }^{14}$ with stage I TTTS defined as polyhydramnios (DVP $\geq 8 \mathrm{~cm}$ ) of one twin, the "recipient," and oligohydramnios (DVP $\leq 2 \mathrm{~cm}$ ) of the cotwin, the "donor." Additional sonographic findings of nonvisualization of the donor twin bladder (stage II), critically abnormal Doppler studies (stage III), hydrops fetalis (stage IV), and death of one or both twins (stage V) defined higher stage TTTS. ${ }^{14}$

Patients were followed with serial USs at our center. In general, patients with stage I TTTS were followed with sonograms at least weekly, or more frequently depending on the concern of the consulting perinatologist. At presentation to our institution, sonographic placental mapping, which is included in our institution's protocol for evaluation of complicated MCDA twins, was performed in most cases. This included targeted color and spectral duplex Doppler interrogation to evaluate for the presence of an A-A anastomosis along the fetal surface of the monochorionic placenta. ${ }^{15-17}$ An A-A anastomosis was confirmed when a vessel was seen with pulsatile bidirectional arterial flow coursing between the cord insertion sites along the fetal surface of the shared placenta.

Percent discordance in EFW [(EFW $\left.\left.{ }^{\text {larger twin }}-\mathrm{EFW}^{\text {smaller twin }}\right) / \mathrm{EFW}^{\text {larger twin }} \times 100\right]$ at diagnosis of stage I TTTS as well as percent birth weight discordance (calculated in a similar manner using actual birth weights) were recorded. Twins were considered discordant at diagnosis if they had percent EFW discordance $>20 \%$. The difference in DVPs between twins at the time of diagnosis was also calculated and recorded for each twin pair $(\triangle \mathrm{DVP}=$ DVPrecipient $-D V P^{\text {donor }}$. FGR was defined as EFW $<10$ percentile for one or both of the twins at any point in the pregnancy. Additionally, if comment was made on the original sonographic interpretation about the donor bladder being noticeably "small," "tiny," "intermittently," or "transiently" visible in the setting of stage I TTTS, this was noted.

The patient database and electronic medical record were used to collect additional information regarding demographic characteristics, treatment interventions, and delivery and neonatal outcomes for those pregnancies delivered at our institution. For patients who ultimately delivered at an outside institution, the FTC staff contacted the patients and outside 
hospital providers to collect information regarding clinical course, delivery, and neonatal outcomes. Pregnancies that ended in termination or dilation and curettage were included in the baseline characteristics but were excluded from survival outcomes and calculations of GA at delivery.

Outcomes of MCDA twin pregnancies with stage I TTTS referred to UCSF between January 2006 to July 2009 were previously submitted to NAFTNET for inclusion in the Emery et al study. ${ }^{13}$ This includes five patients in the present study who were evaluated during that timeframe.

Wilcoxon Rank Sum was used to compare median values of nonparametric continuous variables, and Fisher exact test was used to compare proportions given the relatively smaller size of subgroups in our analyses. All statistics were performed using Stata (version 14.0, StataCorp, College Station, TX). This study was approved by the Committee on Human Research (CHR) at UCSF (IRB Study Number 10-04093). Based on the retrospective and anonymized nature of this study, a waiver of patient consent was granted.

\section{Results}

A total of 373 MCDA twin pregnancies were identified during the study time period. Of these, 39 met the criteria for Quintero stage I TTTS at 16 to 28 weeks GA. Seven of these 39 cases (17.9\%) underwent amnioreduction at the time of stage I TTTS diagnosis for symptomatic polyhydramnios and were excluded. Two additional cases of the $39(5.1 \%)$ elected termination of pregnancy for stage I TTTS, and were also excluded.

The remaining 30 cases of stage I TTTS were expectantly managed and were included in this study. Of these 30 cases, 8 (26.7\%) progressed to higher stage TTTS, including two to stage II, three to stage III, two to stage IV, and one to stage V. Median time to progression was $1.7 \pm 3.2$ weeks (median \pm interquartile range).

Patient demographics based on progression status are shown in Table 1. While maternal age and nulliparous status were not significantly different between the pregnancies that remained stable and those that progressed to $\geq$ stage II TTTS, there was a trend toward earlier GA at diagnosis in the patients who progressed $(18.9 \pm 2.9$ weeks versus $21.4 \pm 3.4$ weeks, $p=$ 0.06). All but one of the included cases was diagnosed with stage I TTTS between 16 and 26 weeks' GA and the remaining case was diagnosed at 27.4 weeks' GA. Those that progressed also delivered at a significantly earlier GA (27.7 \pm 5.8 weeks versus $33.9 \pm 4.9$ weeks, $p=$ $0.04)$. Of those that progressed, $7 / 8$ had a subsequent procedure including placental laser ablation (5), amnioreduction (1), and pregnancy termination (1). The remaining pregnancy had progressed to stage V TTTS and thus did not undergo any additional treatment.

Sonographic characteristics of the study cohort by progression status are shown in Table 2 . Comparing the pregnancies that remained stable to those that progressed to higher stage TTTS, there was no significant difference in the DVP of the recipient twin, $\triangle \mathrm{DVP}$, placental location, percent discordance in EFW, or proportion with EFW discordance $>20 \%$ at the time of diagnosis of stage I TTTS. The donor DVP at diagnosis was lower in the group that progressed $(1.3 \pm 0.4 \mathrm{~cm}$ versus $1.6 \pm 0.5 \mathrm{~cm}, p=0.05)$; however, the small magnitude of 
difference in the measurements is unlikely to be clinically relevant. Additionally, there was no difference in percent birth weight discordance or the rate of marginal or velamentous cord insertions for either twin between the two groups. In the complete cohort, however, marginal or velamentous cord insertion was more frequent in the donor as compared with the recipient twin $(63.3 \%$ versus $30.0 \%, p=0.02)$.

Information regarding evaluation for A-A anastomosis was available for 20/30 pregnancies (66.7\%), including $15 / 22(68.2 \%)$ of those that remained stable and $5 / 8(62.5 \%)$ that progressed. Of the pregnancies that underwent evaluation for A-A anastomosis, A-A was seen in $7 / 15(46.7 \%)$ that remained stable and $1 / 5(20 \%)$ that progressed $(p=0.6)$. Stated differently, of the eight pregnancies with a visible A-A, seven (87.5\%) remained stable.

In 7 of the 30 pregnancies, the urinary bladder in the donor twin was visible on US but was described as being notably "small," "tiny," "intermittently," or "transiently" visible. While not statistically significant, there was a trend toward this observation being associated with progression to higher stage TTTS, with $4 / 7(57.1 \%)$ of these pregnancies progressing $(p=$ $0.06)$.

Pregnancy outcomes are presented in Table 3. Excluding the one termination, overall survival occurred in 47/58 (81.0\%) fetuses, and 26/29 (89.7\%) pregnancies had survival of at least one twin. Patients who remained stable had an increased rate of dual survival (86.4\% versus $28.6 \%, p \leq 0.01)$ and "good" outcome $(77.2 \%$ versus $28.6 \%, p=0.03)$ compared with those that progressed to higher stage TTTS. Progression to higher stage TTTS was associated with an earlier GA at delivery and increased risk of "mixed" or "poor" obstetric outcomes.

\section{Comment}

In our cohort of expectantly managed stage I TTTS pregnancies, three-fourths remained stable and the majority had survival of both twins regardless of progression status. Though not statistically significant, almost $90 \%$ of patients with an identifiable A-A anastomosis remained stable, whereas more than half of those with a small or transient donor bladder progressed to $\geq$ stage II TTTS.

The optimal management of stage I TTTS is controversial, with treatment approaches ranging from expectant management to FLC. Currently, the ideal approach to balance the relative risks of possible progression and procedural complications in stage I TTTS is unclear.

Three prior prospective randomized controlled trials (RCTs) have compared treatment options for TTTS, $5,7,18$ although only the Eurofetus trial-which compared amnioreduction to FLC-reported results for stage I specifically and only six stage I TTTS pregnancies underwent FLC in that trial. ${ }^{5}$ Importantly, none of these trials randomized patients to expectant management. Nonrandomized data regarding outcomes with stage I TTTS is also limited. In a large systematic review of treatment outcomes of stage I TTTS ( $n=262$ twin pairs), Rossi and D'Addario reported that neonatal survival rates were lowest for amnioreduction (77\%) but equivalent for expectant management and FLC (86\% versus 85\%, 
respectively). In a meta-analysis of FLC for stage I TTTS ( $n=60$ twin pairs), overall neonatal survival was similar to expectant management at $83 \% .{ }^{19}$ Furthermore, in a systematic review and meta-analysis of 18 studies $(n=172$ twin pairs to assess for progression, $n=433$ twin pairs to assess for survival), Khalil et al similarly reported a $27 \%$ rate of progression and $79 \%$ overall fetal survival with expectant management of stage I TTTS. ${ }^{20}$

These results are consistent with our findings of a $26 \%$ rate of progression and $72 \%$ overall neonatal survival, and stand in contrast to the dramatically higher rates of progression and significantly worse prognosis reported for expectant management of stage I TTTS in the NAFTNET study. ${ }^{13}$ While the authors of that study endorsed the use of laser for stage I TTTS based on their results, the inherent limitations of the retrospective, nonrandomized study preclude a broad recommendation for intervention for stage I TTTS. Given the risk of iatrogenic procedural complications with FLC, additional evidence is needed to prove the safety and superiority of this technique in stage I TTTS.

While there is inconsistency among studies and controversy regarding management of stage I TTTS, there is consensus that progression is associated with worse outcomes. Therefore, identifying features associated with progression could help stratify risk and might improve outcomes by allowing for better selection of those who would most benefit from surgical intervention. We report two US findings that may serve as such markers: identifiable A-A anastomosis and small/intermittently visible donor bladder.

In our cohort, patients with an A-A anastomosis, identifiable by in utero Doppler US, had only a $12.5 \%$ risk of progression. This is consistent with prior studies that showed A-A anastomoses reduce the risk of developing TTTS in uncomplicated MCDA pregnancies. $15,21,22$ The specificity of prenatal Doppler US to identify A-A anastomoses approaches $100 \%$ but the sensitivity is only 75 to $85 \%$, thus the absence of an A-A anastomosis on US is less informative. ${ }^{23,24} \mathrm{~A}$ notably small or transiently visualized bladder in the donor twin was associated with $>50 \%$ risk of progression to higher stage TTTS. In the widely used Quintero staging system, stage I TTTS specifies visualization of the donor bladder. ${ }^{16}$ This criterion may be variably interpreted and applied (consistently versus transiently visible donor bladder) by investigators, possibly resulting in differences in reported outcomes. Based on our observations, very close, short interval follow-up is performed if the donor twin does not have a consistently visible, normal-appearing bladder for the duration of the sonogram. While these findings did not reach statistical significance with our relatively small sample size, these trends suggest A-A anastomoses and donor bladder appearance warrant further investigation and may be valuable predictors of progression in stage I TTTS.

The lower DVP at diagnosis in the pregnancies that progressed to higher stage TTTS is consistent with the more frequent observation of a small or transiently visualized donor twin bladder in this group. Though the small absolute difference in DVP seen in this study may limit the clinical relevance of this observation, both of these findings suggest a more substantial reduction in the fetal urine production of the donor twin which may indicate greater intertwin hemodynamic imbalance and associated increased risk of progression to severe TTTS. 
Additionally, it is notable that the median GA at diagnosis was nearly 3 weeks earlier in the pregnancies that progressed than those that remained stable. While the data from this cohort is insufficient to define a clear GA cutoff at which the risk of progression significantly increases, our findings suggest that pregnancies diagnosed earlier in gestation may warrant closer US surveillance.

Strengths of this study include consistency of the results with most prior studies of stage I TTTS and information regarding novel predictors of progression. Limitations of this study include the observational, retrospective design of the study and the small number of observations in the subgroups. Additionally, as a tertiary referral center, referral bias is possible and patients with stage I TTTS who remained stable or suffered fetal loss and were not referred may have been missed. Furthermore, while short-term neonatal survival and outcomes were presented, long-term outcomes were unavailable. The lack of placental vascular dye injection studies also limits our ability to confirm the US findings of vascular anastomoses and understand the pathophysiology of TTTS in more detail.

With expectant management of stage I TTTS, three-fourths of patients remain stable and the majority has survival of at least one twin. Findings suggest that A-A anastomoses are not associated with progression, whereas earlier GA at diagnosis or a markedly small or intermittently visible donor bladder may be associated with progression to $\geq$ stage II TTTS. Further research is needed to continue to evaluate the natural history and outcomes of stage I TTTS in a broader, nonreferral population and to refine our understanding of predictors of progression. Prospective RCTs, such as the ongoing international study by Ville et al, ${ }^{25}$ comparing expectant management versus FLC for stage I TTTS, are needed to more definitely determine the optimal strategy for management of stage I TTTS.

\section{Acknowledgments}

\section{Funding}

Dr. Sparks was supported by grant 5K12HD001262-18 from the National Institutes of Health (NIH). The contents of the publication are solely the responsibility of the authors and do not necessarily represent the official views of the NIH.

\section{References}

1. Acosta-Rojas R, Becker J, Munoz-Abellana B, Ruiz C, Carreras E, Gratacos E. Catalunya and Balears Monochorionic Network. Twin chorionicity and the risk of adverse perinatal outcome. Int J Gynaecol Obstet. 2007; 96(02):98-102. [PubMed: 17250837]

2. Lewi L, Jani J, Blickstein I, et al. The outcome of monochorionic diamniotic twin gestations in the era of invasive fetal therapy: a prospective cohort study. Am J Obstet Gynecol. 2008; 199(05): 514.e1-514.e8. [PubMed: 18533114]

3. Gul A, Aslan H, Polat I, et al. Natural history of 11 cases of twin-twin transfusion syndrome without intervention. Twin Res. 2003; 6(04):263-266. [PubMed: 14511429]

4. Berghella V, Kaufmann M. Natural history of twin-twin transfusion syndrome. J Reprod Med. 2001; 46(05):480-484. [PubMed: 11396376]

5. Senat MV, Deprest J, Boulvain M, Paupe A, Winer N, Ville Y. Endoscopic laser surgery versus serial amnioreduction for severe twin-to-twin transfusion syndrome. N Engl J Med. 2004; 351(02): 136-144. [PubMed: 15238624] 
6. Rossi AC, D’Addario V. Laser therapy and serial amnioreduction as treatment for twin-twin transfusion syndrome: a meta-analysis and review of literature. Am J Obstet Gynecol. 2008; 198(02):147-152. [PubMed: 18068144]

7. Crombleholme TM, Shera D, Lee H, et al. A prospective, randomized, multicenter trial of amnioreduction vs selective fetoscopic laser photocoagulation for the treatment of severe twin-twin transfusion syndrome. Am J Obstet Gynecol. 2007; 197(04):396.e1-396.e9. [PubMed: 17904975]

8. Roberts D, Neilson JP, Kilby MD, Gates S. Interventions for the treatment of twin-twin transfusion syndrome. Cochrane Database Syst Rev. 2014:CD002073(01)-CD002073. [PubMed: 24482008]

9. Simpson LL. Society for Maternal-Fetal Medicine. Twin-twin transfusion syndrome. Am J Obstet Gynecol. 2013; 208(01):3-18. [PubMed: 23200164]

10. Bebbington MW, Tiblad E, Huesler-Charles M, Wilson RD, Mann SE, Johnson MP. Outcomes in a cohort of patients with Stage I twin-to-twin transfusion syndrome. Ultrasound Obstet Gynecol. 2010; 36(01):48-51. [PubMed: 20201111]

11. Meriki N, Smoleniec J, Challis D, Welsh AW. Immediate outcome of twin-twin transfusion syndrome following selective laser photocoagulation of communicating vessels at the NSW Fetal Therapy Centre. Aust N Z J Obstet Gynaecol. 2010; 50(02):112-119. [PubMed: 20522065]

12. Rossi AC, D'Addario V. Survival outcomes of twin-twin transfusion syndrome stage I: a systematic review of literature. Am J Perinatol. 2013; 30(01):5-10. [PubMed: 22836822]

13. Emery SP, Hasley SK, Catov JM, et al. North American Fetal Therapy Network. North American Fetal Therapy Network: intervention vs expectant management for stage I twin-twin transfusion syndrome. Am J Obstet Gynecol. 2016; 215(03):346.e1-346. e7. [PubMed: 27131587]

14. Quintero RA, Morales WJ, Allen MH, Bornick PW, Johnson PK, Kruger M. Staging of twin-twin transfusion syndrome. J Perinatol. 1999; 19(8 Pt 1):550-555. [PubMed: 10645517]

15. Denbow ML, Cox P, Talbert D, Fisk NM. Colour Doppler energy insonation of placental vasculature in monochorionic twins: absent arterio-arterial anastomoses in association with twinto-twin transfusion syndrome. Br J Obstet Gynaecol. 1998; 105(07):760-765. [PubMed: 9692418]

16. Erskine RL, Ritchie JW, Murnaghan GA. Antenatal diagnosis of placental anastomosis in a twin pregnancy using Doppler ultrasound. Br J Obstet Gynaecol. 1986; 93(09):955-959. [PubMed: 3533136]

17. Hecher K, Jauniaux E, Campbell S, Deane C, Nicolaides K. Artery-to-artery anastomosis in monochorionic twins. Am J Obstet Gynecol. 1994; 171(02):570-572. [PubMed: 8059848]

18. Moise KJ Jr, Dorman K, Lamvu G, et al. A randomized trial of amnioreduction versus septostomy in the treatment of twin-twin transfusion syndrome. Am J Obstet Gynecol. 2005; 193(3 Pt 1):701707. [PubMed: 16150263]

19. Rossi AC, D'Addario V. The efficacy of Quintero staging system to assess severity of twin-twin transfusion syndrome treated with laser therapy: a systematic review with meta-analysis. Am J Perinatol. 2009; 26(07):537-544. DOI: 10.1055/s-0029-1215430 [PubMed: 19283655]

20. Khalil A, Cooper E, Townsend R, Thilaganathan B. Evolution of stage 1 twin-to-twin transfusion syndrome (TTTS): systematic review and meta-Analysis. Twin Res Hum Genet. 2016; 19(03): 207-216. [PubMed: 27137946]

21. Denbow ML, Cox P, Taylor M, Hammal DM, Fisk NM. Placental angioarchitecture in monochorionic twin pregnancies: relationship to fetal growth, fetofetal transfusion syndrome, and pregnancy outcome. Am J Obstet Gynecol. 2000; 182(02):417-426. [PubMed: 10694346]

22. de Villiers SF, Slaghekke F, Middeldorp JM, Walther FJ, Oepkes D, Lopriore E. Arterio-arterial vascular anastomoses in monochorionic placentas with and without twin-twin transfusion syndrome. Placenta. 2012; 33(08):652-654. [PubMed: 22652047]

23. Fichera A, Mor E, Soregaroli M, Frusca T. Antenatal detection of arterio-arterial anastomoses by Doppler placental assessment in monochorionic twin pregnancies. Fetal Diagn Ther. 2005; 20(06): 519-523. [PubMed: 16260888]

24. Taylor MJ, Denbow ML, Tanawattanacharoen S, Gannon C, Cox PM, Fisk NM. Doppler detection of arterio-arterial anastomoses in monochorionic twins: feasibility and clinical application. Hum Reprod. 2000; 15(07):1632-1636. [PubMed: 10875880] 
25. [Accessed January 29, 2018] A cluster randomized trial comparing a conservative management and primary laser surgery. Available at: https://clinicaltrials.gov/ct2/show/study/NCT01220011?

term=twin-twin+transfusion+syndrome \&rank=9 


\section{Table 1}

Patient characteristics

\begin{tabular}{|c|c|c|c|c|}
\hline \multirow[b]{2}{*}{ Variable } & \multirow[b]{2}{*}{ All patients $(n=30)$} & \multicolumn{3}{|c|}{ By progression to higher stage TTTS } \\
\hline & & No progression $(n=22)$ & Progression $(n=8)$ & $p$-Value \\
\hline Maternal age, $\mathrm{y}^{a}$ & $30(10.0)$ & $30(10.0)$ & $30.5(10.0)$ & 0.87 \\
\hline Nulliparity, $n(\%)^{b}$ & $14(46.7)$ & $12(54.6)$ & $2(25.0)$ & 0.23 \\
\hline GA at diagnosis, wk ${ }^{a}$ & $20.8(3.7)$ & $21.4(3.4)$ & $18.9(2.9)$ & 0.06 \\
\hline GA at delivery, $\mathrm{wk}^{a}$ & $31.4(7.2)$ & $33.9(4.9)$ & $27.7(5.8)$ & 0.04 \\
\hline \multicolumn{4}{|l|}{ Mode of delivery $b$} & \multirow[t]{4}{*}{0.09} \\
\hline $\mathrm{SVD}, n(\%)$ & $9(30.0)$ & $5(22.7)$ & $4(50.0)$ & \\
\hline C section, $n(\%)$ & $20(64.3)$ & $17(77.3)$ & $3(37.5)$ & \\
\hline Elective termination, $n(\%)$ & $1(3.3)$ & $0(0.0)$ & $1(12.5)$ & \\
\hline
\end{tabular}

Abbreviations: GA, gestational age; SVD, spontaneous vaginal delivery; TTTS, twin-twin transfusion syndrome.

Note: $N$ values represent number of pregnancies. Stage I TTTS groups with and without progression to higher stage TTTS are the comparison groups for the $p$-values. Wilcoxon Rank Sum and Fisher exact tests were used to compare nonparametric continuous variables and proportions, respectively. A significance level of 0.05 was used to define significance for these analyses.

${ }^{a}$ Results for continuous variables are displayed as median (interquartile range).

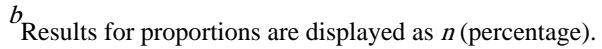


Table 2

Sonographic characteristics

\begin{tabular}{|c|c|c|c|c|}
\hline \multirow[b]{2}{*}{ Variable } & \multirow[b]{2}{*}{ All patients $(n=30)$} & \multicolumn{3}{|c|}{ By progression to higher stage TTTS } \\
\hline & & $\begin{array}{l}\text { No progression }(n= \\
\text { 22) }\end{array}$ & Progression $(n=8)$ & $p$-Value \\
\hline DVP of recipient twin, $\mathrm{cm}^{a, b}$ & $9.6(1.6)$ & $9.6(1.7)$ & $9.7(1.2)$ & 0.83 \\
\hline DVP of donor, $\mathrm{cm}^{a, b}$ & $1.4(0.4)$ & $1.6(0.5)$ & $1.3(0.4)$ & 0.05 \\
\hline$\Delta \mathrm{DVP}, \mathrm{cm}^{a, b}$ & $8.3(1.6)$ & $8.1(1.7)$ & $8.3(1.5)$ & 0.89 \\
\hline EFW discordance, $\%^{a, b}$ & $18.0(17.0)$ & $19.1(12.0)$ & $12.5(23.1)$ & 0.61 \\
\hline EFW discordance $>20 \%, n(\%) b, c$ & $13(43.3)$ & $10(45.5)$ & $3(37.5)$ & 1.0 \\
\hline Birth weight discordance, $\%^{a}$ & $\begin{array}{l}20 / 22 \\
19.8(22.6)\end{array}$ & $\begin{array}{l}17 / 18 \\
22.3(21.2)\end{array}$ & $\begin{array}{l}3 / 4 \\
15.9(29.6)\end{array}$ & 0.31 \\
\hline FGR of at least one twin, $n(\%)^{a, d}$ & $1(3.6)$ & $1(4.8)$ & $0(0.0)$ & 1.0 \\
\hline A-A anastomosis interrogated, $n$ & $20 / 30$ & $15 / 22$ & $5 / 8$ & \multirow[t]{2}{*}{0.60} \\
\hline A-A anastomosis identified, $n(\%)^{\mathcal{C}}$ & $8(40.0)$ & $7(46.7)$ & $1(20.0)$ & \\
\hline Marginal/velamentous cord insertion of recipient, $n(\%)^{c}$ & $9(30.0)$ & $5(22.7)$ & $4(50.0)$ & 0.20 \\
\hline Marginal/velamentous cord insertion of donor, $n(\%)^{c}$ & $19(63.3)$ & $14(63.6)$ & $5(62.5)$ & 1.0 \\
\hline \multicolumn{4}{|l|}{ Placental location, $n(\%)^{\mathcal{C}}$} & \multirow[t]{3}{*}{1.0} \\
\hline Anterior & $15(50.0)$ & $11(50.0)$ & $4(50.0)$ & \\
\hline Posterior & $15(50.0)$ & $11(50.0)$ & $4(50.0)$ & \\
\hline Donor twin bladder "small" or "intermittent," $n(\%)^{c}$ & $7(23.3)$ & $3(13.6)$ & $4(50.0)$ & 0.06 \\
\hline
\end{tabular}

Abbreviations: A-A, arterio-arterial anastomosis; DVP, deepest vertical pocket; EFW, estimated fetal weight; FGR, fetal growth restriction; TTTS, twin-twin transfusion syndrome.

Note: $N$ values represent number of pregnancies. Stage I TTTS groups with and without progression to higher stage TTTS are the comparison groups for the $p$-values. Wilcoxon Rank Sum and Fisher exact tests were used to compare nonparametric continuous variables and proportions, respectively. A significance level of 0.05 was used to define significance for these analyses.

${ }^{a}$ Results for continuous variables are displayed as median (interquartile range).

$b_{\text {At diagnosis of stage I TTTS. }}$

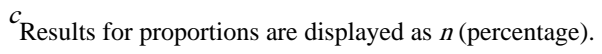

${ }^{d}$ Defined as EFW $\leq 10$ percentile for GA for either twin at any time from diagnosis of stage I TTTS to delivery. 
Table 3

\section{Pregnancy outcomes}

\begin{tabular}{|l|l|l|l|l|}
\hline & \multirow{2}{*}{} & \multirow{2}{*}{ All patients $(\boldsymbol{n}=\mathbf{2 9})^{\boldsymbol{a}}$} & \multicolumn{2}{l|}{ By progression to higher stage TTTS } \\
\cline { 5 - 6 } & & No progression $(\boldsymbol{n}=\mathbf{2 2})$ & ${\text { Progression }(\boldsymbol{n}=\mathbf{7})^{\boldsymbol{a}}}^{\boldsymbol{a}} \boldsymbol{p}$-value \\
\hline Survivors per pregnancy, $\boldsymbol{n}(\%)$ & & & & $<$ \\
\hline 2 & $21(72.4)$ & $19(86.4)$ & $2(28.6)$ & \\
\hline 1 & $5(17.2)$ & $1(4.6)$ & $4(57.1)$ & \\
\hline 0 & $3(10.3)$ & $2(9.1)^{b}$ & $1(14.3)^{c}$ & \\
\hline$\geq 1$ & $26(89.7)$ & $20(90.9)$ & $6(85.7)$ & \\
\hline Outcome, $\boldsymbol{n}(\%)^{d}$ & & & & $\mathbf{0 . 0 4}$ \\
\hline Good & $19(65.5)$ & $17(77.2)$ & $2(28.6)$ & \\
\hline Mixed & $5(17.2)$ & $3(13.6)$ & $2(28.6)$ & \\
\hline Poor & $5(17.2)$ & $2(9.1)^{b}$ & $3(42.9)^{c}$ & \\
\hline
\end{tabular}

Abbreviation: TTTS, twin-twin transfusion syndrome.

Note: $N$ values represent number of pregnancies. Stage I TTTS groups with and without progression to higher stage TTTS are the comparison groups for the $p$-values. A significance level of 0.05 was used to define significance for these analyses.

a Excluding patient who underwent elective termination for stage III TTTS.

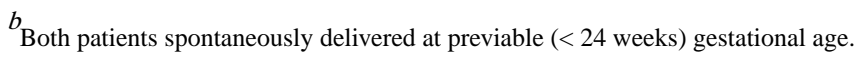

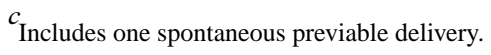

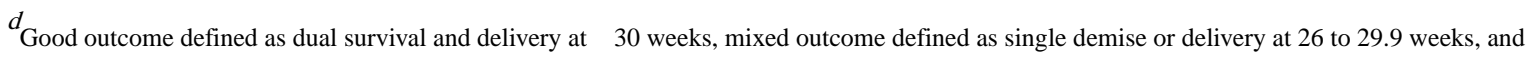
poor outcome defined as double demise or delivery at less than 26 weeks. 